\title{
PERIPHERAL NERVE DYSFUNCTION AMONG WORKERS WITH LONG-TERM OCCUPATIONAL EXPOSURE TO ORGANOPHOSPHATE PESTICIDES.
}

\author{
By \\ El-Helaly ME., ${ }^{*}$ Hazem M., ${ }^{* *}$ Mosad SM., ${ }^{* * *}$ El- Biomy AA..**** \\ From \\ ${ }^{*}$ Department of Community Medicine, ${ }^{* *}$ Department of Neurology, \\ ${ }^{* * *}$ Department of Forensic Medicine and Clinical Toxicology, \\ ${ }^{* * * *}$ Department of Clinical Pathology, Faculty of Medicine. Mansoura University, Mansoura, Egypt
}

\begin{abstract}
:
A cross-sectional study was carried out to assess cholinesterase activity, study the work practices and investigate the prevalence of peripheral nerve dysfunction among workers with long-term exposure to Organophosphate pesticides (OP). The study was carried out on 36 Organophosphate sprayers with long-term exposure to Organophosphate and 20 controls unexposed to pesticides, from Dekernes, Dakahlia Governorate, Egypt. All participant were submitted to general medical examination, neurological evaluation, nerve conduction study and electromyography. Plasma cholinesterase (AChE) level was estimated for both OP sprayers and the controls. Most of the OP sprayers had practiced pesticide application with improper personal hygiene. OP sprayers had a statistically significant lower plasma AChE mean level $(1548.9 \pm 801.7 \mathrm{mU} /$ $\mathrm{ml})$ compared to the controls $(6751.7 \pm 990.8 \mathrm{mU} / \mathrm{ml})$. The study reported a higher prevalence of peripheral neuropathies among OP sprayers $(38.9 \%)$ in comparison to the controls $(5.0 \%)(\mathrm{P}<0.01)$. However, most of the diagnosed neuropathies among the OP sprayers were subclinical neuropathies (78.6\%) and possible neuropathies (21.4\%). In conclusion, long-term occupational exposure to $\mathrm{OP}$, was associated with subclinical and possible neuropathies, but not confirmed neuropathies .These results may reflect an early stage of OP toxic effects on the peripheral nerves among Organophosphate sprayers due to chronic exposure.
\end{abstract}

Keyword: Organophosphate, neuropathy, pesticides.

Abbreviations: AChE, acetylcholinesterase; BMI, body mass index; NCS, nerve conduction studies, OPIDP,organophosphate induced delayed polyneuropathy; OP, organophosphate; QST, quantitative sensory testing. 
El-Helaly ME. et al.,

\section{Introduction}

Organophosphate pesticides (OP) are phosphoric acid esters or thiophosphoric acid esters and are among the most widely used pesticides. Several organophosphorus esters are used as insecticides because they inhibit the acetylcholinesterase (AChE) of insects. The same mechanism accounts for acute toxicity in humans and is characterized by signs of cholinergic overstimulation (Cope et al., 2004). In addition, certain Organophosphate may cause a distal, sensory-motor, central-peripheral axonopathy known as organophosphate-induced delayed polyneuropathy (OPIDP) following severe poisoning (Moretto and Lotti, 1998). OPIDP is a dying back axonopathy characterised by sensory and motor symptoms that develop days to weeks after poisoning. Among the OP repeatedly reported to have caused neuropathy in humans are methamidophos, chlorpyrifos, and trichlorphon. It has been suggested that persistent subclinical neuropathy may be frequent (Miranda et al.,2004).

Recent studies suggest that Organophosphate (OP) can produce long-term effects which may be secondary to one or more acute toxic episodes or as a result of chronic long-term exposure to low doses of OP insufficient to produce acute symptoms (Jamal et al., 2002a ; Kamel et al.,
2005). So, The neurotoxic consequences of acute high - level OP exposure are well established but whether exposure to more moderate levels of OP is also neurotoxic is more controversial and needs more research (Kamel et al., 2007). The aim of the study was to, assess cholinesterase activity, study the work practices and investigate the prevalence of peripheral nerve dysfunction among workers with long-term exposure to Organophosphate pesticides.

\section{Methods}

This is a comparative cross-sectional study which was carried out on workers with long-term exposure to OP (OP sprayers) and controls unexposed to pesticides, from Dekernes, Dakahlia Governorate, Egypt; where OP were widely used in a large number of fields. All participants in this study were volunteers who signed a statement of formal consent before testing. Thirty six males (OP sprayers) were recruited from those who were working in the pesticide application departments at Dekernes district, Dakahlia Governorate, Egypt ( $\mathrm{n}=48$ males). Among the 48 workers, $9(18.8 \%)$ were excluded and 3 ( $6.2 \%)$ refused to participate, so the overall response rate was $75.0 \%$. Their ages ranged from 22 to 63 years with a mean value of $47.55 \pm 12.33$ years. OP sprayers worked 6 - 10 weeks/ season for a duration ranging 
from 4 to 17 years with a mean of 9.44 \pm 4.21 years. A typical workday was from 8.00 am to 12.00 noon, then from $3.00 \mathrm{pm}$ to $7.00 \mathrm{pm}$, six days per week. They were involved in OP spraying on almost all crops and orchards found in Egypt. The different types of pesticides sprayed by the workers were identified by an agriculture engineer and are listed in table 1 , according to WHO recommended classification of pesticides by hazard (WHO, 2004). The pesticides were applied by Knapsack sprayers (carrying 120 liters of pesticides) or motorized sprayers such as tractors (400- 600 liters). The exposure routes for all OP sprayers were inhalation, skin contact and oral routes. Thirty three OP sprayers reported that they had never used protective equipment while three OP sprayers used only ordinary masks. The reasons of not using protective equipment were lack of economic resources as reported by 30 workers $(83.3 \%)$ and because of inconvenience as reported by 6 workers $(16.7 \%)$, however all of the workers knew the importance of the protective equipment. A control group consisting of 20 males were recruited from sanitation workers from different schools at Dekernes. They had never been exposed to pesticides in their work, with no evident occupational or non-occupational exposures to Pesticide. The controls matched the OP sprayers group in age, Body Mass
Index (BMI), marital state, educational level, smoking habit and duration of employment.

Exclusion Criteria for this study were medical diseases including diabetes mellitus, liver diseases, renal diseases, vitamin deficiency, and recent exposure to other neurotoxic agents. Workers were excluded if they were seasonal workers or had an acute OP poisoning attack, one month from the examination. Participants were interviewed using a standardized questionnaire which included questions about a) personal history, b) family and past history specially of neurological diseases, c) occupational history including questions about work practices, personal hygiene and use of protective equipment during pesticide application, also trade names of used pesticides (Niven et al., 1993), d) environmental history of chemical exposures at home and possible non-occupational exposures to pesticides, and e) personal medical history including peripheral nervous system evaluation. General medical examinations were performed to detect medical conditions that potentially produce nervous system abnormalities. The peripheral nervous system examination included evaluation of strength (proximal and distal); gait; muscle stretch reflexes (wrist, knee, and ankle); and sensation (fine touch, Von Frey mono- 
filament, joint position sensation, pin pain, and vibration). Sensation was tested at the dominant index finger and big toe, and, if abnormal, contralaterally.

Nerve conduction studies (NCS) were carried out with standard techniques according to Oh (2003), on both right and left sides. In motor conduction studies of median, ulnar, tibial and common peroneal nerves; terminal latency, amplitude of compound muscle action potential, motor nerve conduction velocity were measured. In the sensory studies of median, ulnar and superficial peroneal nerves; terminal latency, amplitude of sensory nerve action potential, and sensory nerve conduction velocity were measured. NCS were done in the Electrophysiology Unit at the Neurology Department, Mansoura University Hospital; using Neuropack 2 device, Nihonkohden, model MEB/MEM. 7102 A/K.02, Tokyo, Japan. Regarding, Electromyography (EMG), action potentials for muscles were studied using standard concentric needle. Neuropathy outcome variables were diagnosed as follow: subclinical or clinically evident neuropathy was defined using a combination of symptoms, signs, and NCS results as consistent with standard clinical practice (Dyck et al., 1995; Albers et al., 2004). Diagnostic classification of neuropathy included: subclinical, possible, probable and confirmed neuropathy.
AChE measurement: a sample of five milliliters of venous blood was taken under complete aseptic precautions from each participant. Blood samples were left to clot , plasma was analyzed for acetyl cholinesterase activity (AChE) by kinetic determination using butyril thiocholine dithiobin nitrobenzoic acid method (Ellman et al., 1961). The material was supplied by Biostc (Italy), in which acetylcholine catalyzed the hydrolysis of butyrithiocholine forming butyrate and thiocholine. Thiocholine reacts with dithiobin nitrobenzoic acid forming a colored compound.

The results were statistically analysed using chi-square test $\left(\chi^{2}\right)$ and Fisher exact test (for qualitative data); and student t-test, ANOVA (for parametric quantitative data). Mann-Whitney test and Kruskal Wallis Test were used for nonparametric quantitative data. Analysis of data was performed using the 0.05 significance level and the 0.01 high significance level.

\section{Results}

There was no statistically significant difference between the OP sprayers and the controls as regard matching variables of age, height, weight (BMI) smoking habit, educational level, marital status and duration of employment (table 1). Moreover, all study population was of rural residence. 
Malathion was sprayed by all OP sprayers (100\%) followed by Dimethoate $(97.2 \%)$ and Fenthion (83.3). However, only $27.8 \%$ and $13.9 \%$ sprayed Chlorpyrifos and Quinalphas, respectively (table 2). Moreover, all of the sprayed OP at the present study were classified by WHO (2004) as moderately hazardous Pesticides except Malathion which is classified as slightly hazardous. Most of the OP sprayers had practiced OP application with improper personal hygiene, concerning: storage, mixing and preparation, spraying, disposal, and afterwork cleaning up $(88.9,100.0,86.1,94.4$, $88.9 \%$, respectively) (table 3 ). OP sprayers had a lower plasma AChE mean level $(1548.9 \pm 801.7 \mathrm{mU} / \mathrm{ml})$ compared to that of the controls $(6751.7 \pm 990.8 \mathrm{mU} / \mathrm{ml})(\mathrm{P}<$ 0.01) (Tab. 4).

Among OP sprayers, 8.3\% had symptoms of neuropathy which none of the controls had symptoms, the difference was not statistically significant. Concerning signs of neuropathy among OP sprayers, 8.3\% had lower limb sensory signs, $2.7 \%$ had knee hyporeflexia and 5.6\% had ankle hyporeflexia, while none of the controls had signs; the difference was not statistically significant. Among OP sprayers, lower limb sensory signs were more prevalent $(8.3 \%)$ compared to those of the upper limbs $(2.7 \%)$. Also, knee and ankle hyporeflexia were more prevalent $(2.78 \%$ and $5.6 \%$, respectively) compared to wrist hyporeflexia $(0 \%)$. However, there was no motor signs in the upper limb among both OP sprayers and the controls (table 5).

Concerning motor NCS, all the upper limbs nerves examined among OP sprayers showed prolonged terminal latencies and lower amplitudes compared to those of the controls $(\mathrm{P}<0.01)$. However, motor conduction velocities did not differ significantly between both groups except for the right ulnar nerve which showed slower motor conduction velocities among OP sprayers compared to those of the controls $(\mathrm{P}<0.01)$. Regarding motor NCS of the lower limbs, all examined nerves among OP sprayers showed lower amplitudes and slower motor conduction velocities in comparison to those of the controls $(\mathrm{P}<0.01)$. However, terminal latencies did not show any significant difference between both groups (table 6). Regarding sensory NCS, results of the upper limbs nerves examined did not differ significantly between both groups. However, amplitudes of right and left median and right ulnar nerves among OP sprayers were significantly lower than those of the controls. Moreover, the terminal latency of the right ulnar nerve among OP sprayers were more prolonged than those of the controls $(\mathrm{P}<0.05)($ table 7). 
El-Helaly ME. et al.,

On the other hand, all the lower limbs nerves examined among OP sprayers showed prolonged terminal latencies, lower amplitudes and slower sensory conduction velocities in comparison to those of the controls $(P<0.01)$. Peripheral neuropathies were found to be more prevalent among $\mathrm{OP}$ sprayers $(38.9 \%)$ compared to the controls $(5.0 \%)$ with a high statistically significant difference $(\mathrm{P}<0.01)$ (table 8$)$. All the diagnosed neuropathies among the OP sprayers were classified as subclinical neuropathies $(78.6 \%)$ and possible neuropathies
(21.4\%) with no probable nor confirmed neuropathies. Moreover, all neuropathies were sensory-motor polyneuropathies, distributed distal more than proximal. On the other hand, only one case was diagnosed as subclinical mononeuropathy among the controls (table 9).

OP sprayers with neuropathy had lower mean of AChE levels $(\mathrm{P}<0.01)$ and longer mean duration of employment $(\mathrm{P}<0.05)$ in comparison to OP sprayers without neuropathy. However, the age did not differ significantly (table 10). 
Table 1. Demographic characteristics of OP sprayers and the controls.

\begin{tabular}{|c|c|c|c|c|c|c|}
\hline \multirow[t]{2}{*}{ Variables } & \multicolumn{2}{|c|}{$\begin{array}{c}\text { OP sprayers } \\
n=36\end{array}$} & \multicolumn{2}{|c|}{$\begin{array}{c}\text { Controls } \\
n=20\end{array}$} & \multirow{2}{*}{$\begin{array}{c}\text { Tests of } \\
\text { significance }\end{array}$} & \multirow{2}{*}{$\begin{array}{c}\mathbf{P} \\
\text { Value }\end{array}$} \\
\hline & Mean & $\pm \mathrm{SD}$ & Mean & $\pm \mathrm{SD}$ & & \\
\hline Age ( year) & 47.55 & 12.33 & 47.50 & 7.57 & ${ }^{\mathrm{a}} \mathrm{t}=0.01$ & $>0.05$ \\
\hline Height ( m) & 168.33 & 6.12 & 169.60 & 6.59 & $\mathrm{t}=0.72$ & $>0.05$ \\
\hline Weight ( kg) & 73.83 & 11.85 & 78.10 & 9.88 & $\mathrm{t}=1.36$ & $>0.05$ \\
\hline \multirow[t]{2}{*}{ BMI } & 25.89 & 2.55 & 27.06 & 2.06 & $\mathrm{t}=1.74$ & $>0.05$ \\
\hline & $\mathrm{n}$ & $(\%)$ & $\mathrm{n}$ & $(\%)$ & & \\
\hline $\begin{array}{l}\text { Smoking } \\
\text {-Smoker } \\
\text {-Non-smoker }\end{array}$ & $\begin{array}{l}20 \\
16\end{array}$ & $\begin{array}{l}55.6 \\
44.4\end{array}$ & $\begin{array}{c}14 \\
6\end{array}$ & $\begin{array}{l}70.0 \\
30.0\end{array}$ & ${ }^{\mathrm{b}} \chi^{2}=1.12$ & $>0.05$ \\
\hline $\begin{array}{l}\text { Education } \\
\text {-Illiterate } \\
\text {-Elementary school } \\
\text {-Diploma }\end{array}$ & $\begin{array}{c}16 \\
14 \\
6\end{array}$ & $\begin{array}{l}44.4 \\
38.9 \\
16.7\end{array}$ & $\begin{array}{c}7 \\
10 \\
3\end{array}$ & $\begin{array}{l}35.0 \\
50.0 \\
15.0\end{array}$ & $\chi^{2}=0.67$ & $>0.05$ \\
\hline $\begin{array}{l}\text { Marital status: } \\
\text {-Married } \\
\text {-Single }\end{array}$ & $\begin{array}{c}32 \\
4\end{array}$ & $\begin{array}{l}88.9 \\
11.1\end{array}$ & $\begin{array}{c}16 \\
4\end{array}$ & $\begin{array}{l}80.0 \\
20.0\end{array}$ & $\chi^{2}=0.83$ & $>0.05$ \\
\hline Duration of employment (years) & 9.44 & 4.21 & 8.50 & 2.35 & $\mathrm{t}=0.92$ & $>0.05$ \\
\hline
\end{tabular}

a: student-t test

b: chi square test. 
El-Helaly ME. et al.,

Table 2. Percents of workers spraying different types of OP:

\begin{tabular}{|c|c|}
\hline OP Chemical name & $\begin{array}{c}\text { OP sprayers } \\
\text { n }(\%)\end{array}$ \\
\hline $\begin{array}{ll}\text { 1- } & \text { Dimethoate } \\
\text { 2- } & \text { Quinalphas } \\
\text { 3- } & \text { Chlorpyrifos } \\
\text { 4- } & \text { Fenthion } \\
\text { 5- } & \text { Malathion }\end{array}$ & $\begin{array}{ll}35 & (97.2) \\
5 & (13.9) \\
10 & (27.8) \\
30 & (83.3) \\
36 & (100.0)\end{array}$ \\
\hline
\end{tabular}

Table 3. Prevalence of proper and improper work practices among OP sprayers.

\begin{tabular}{|c|c|c|c|c|c|}
\hline \multirow{3}{*}{ Variable } & \multicolumn{4}{|c|}{$\begin{array}{c}\text { Work practices among OP } \\
\text { sprayers }\end{array}$} & \multirow{3}{*}{$\begin{array}{c}\text { Total } \\
\text { n }(\%)\end{array}$} \\
\hline & \multirow{2}{*}{\multicolumn{2}{|c|}{$\begin{array}{l}\text { Proper } \\
\text { n } \%\end{array}$}} & \multicolumn{2}{|c|}{ Improper } & \\
\hline & & & $\mathbf{n}$ & $\%$ & \\
\hline OP Storage & 4 & 11.1 & 32 & 88.9 & $36(100 \%)$ \\
\hline OP mixing and preparation & 0 & 0.0 & 36 & 100.0 & $36(100 \%)$ \\
\hline OP Spraying & 5 & 13.9 & 31 & 86.1 & $36(100 \%)$ \\
\hline OP Disposal & 2 & 5.6 & 34 & 94.4 & $36(100 \%)$ \\
\hline After-work cleaning up & 4 & 11.1 & 32 & 88.9 & $36(100 \%)$ \\
\hline
\end{tabular}


Table 4. Comparison of the means of AChE levels between OP sprayers and the controls.

\begin{tabular}{|l|c|c|c|c|}
\hline \multicolumn{1}{|c|}{ AChE $(\mathbf{m U} / \mathbf{m l})$} & $\begin{array}{c}\text { OP sprayers } \\
\mathbf{n = 3 6}\end{array}$ & $\begin{array}{c}\text { Controls } \\
\mathbf{n = 2 0}\end{array}$ & $\begin{array}{c}\text { Tests of } \\
\text { significance }\end{array}$ & $\begin{array}{c}\text { P } \\
\text { Value }\end{array}$ \\
\hline Mean \pm SD & $1548.9 \pm 801.7$ & $6751.7 \pm 990.8$ & $Z^{*}=6.16$ & $<0.01$ \\
Range & $520.0-2691.0$ & $2691.0-8420.0$ & \\
\hline
\end{tabular}

$\mathrm{Z}=$ Mann-Whitney test

Table 5. Comparison of neurological evaluation parameters suggestive of peripheral neuropathy in OP sprayers and the controls.

\begin{tabular}{|c|c|c|c|c|c|c|}
\hline \multirow{2}{*}{$\begin{array}{c}\text { Neurological } \\
\text { evaluation parameters }\end{array}$} & \multicolumn{2}{|c|}{$\begin{array}{c}\text { OP sprayers } \\
n=36\end{array}$} & \multicolumn{2}{|c|}{$\begin{array}{c}\text { Controls } \\
\mathbf{n}=\mathbf{2 0}\end{array}$} & \multirow{2}{*}{$\begin{array}{c}\text { Test of } \\
\text { significance } \\
\text { (Fisher Exact } \\
\text { Test) }\end{array}$} & \multirow{2}{*}{$\begin{array}{c}\mathbf{P} \\
\text { Value }\end{array}$} \\
\hline & $\mathbf{n}$ & $\%$ & $\mathbf{n}$ & $\%$ & & \\
\hline Symptoms ${ }^{a}$ of neuropathy & 3 & 8.3 & 0 & 0.0 & 1.761 & $>0.05$ \\
\hline \multicolumn{7}{|l|}{ Signs of neuropathy } \\
\hline \multicolumn{7}{|l|}{ Motor signs : } \\
\hline -Upper limb weakness & 0 & 0 & 0 & 0.0 & - & - \\
\hline -Lower limb weakness & 1 & 2.7 & 1 & 5.0 & 0.18 & $>0.05$ \\
\hline \multicolumn{7}{|l|}{ Sensory signs ${ }^{\mathrm{b}}$} \\
\hline -Upper limb affection & 1 & 2.7 & 1 & 5.0 & 0.18 & $>0.05$ \\
\hline -Lower limb affection & 3 & 8.3 & 0 & 0.0 & 1.76 & $>0.05$ \\
\hline \multicolumn{7}{|l|}{ Deep reflexes : } \\
\hline - Wrist joint hyporeflexia & 0 & 0.0 & 0 & 0.0 & - & - \\
\hline - Knee joint hyporeflexia & 1 & 2.7 & 0 & 0.0 & 0.56 & $>0.05$ \\
\hline - Ankle joint hyporeflexia & 2 & 5.6 & 0 & 0.0 & 1.15 & $>0.05$ \\
\hline
\end{tabular}

a: Symptoms of neuropathy: symmetric stocking or stocking-glove distribution numbness, tingling, or sensory loss.

b: Sensory signs include: Pin-point, vibration, joint position, fine touch and touch pressure sensation. N.B. only the symmetrical neurological symptoms and signs were included. 
Table 6. Comparison of Motor Nerve Conduction Study (NCS) results between OP sprayers and the controls.

\begin{tabular}{|c|c|c|c|c|c|c|}
\hline \multirow{2}{*}{ NCS Results } & \multicolumn{2}{|c|}{$\begin{array}{c}\text { OP sprayers } \\
n=36\end{array}$} & \multicolumn{2}{|c|}{$\begin{array}{l}\text { Controls } \\
(n=20)\end{array}$} & \multirow{2}{*}{$\begin{array}{c}\text { Test of } \\
\text { significance } \\
\text { (Student-t test) }\end{array}$} & \multirow{2}{*}{$\begin{array}{c}\mathbf{P} \\
\text { Value }\end{array}$} \\
\hline & Mean & $\pm \mathrm{SD}$ & Mean & $\pm \mathrm{SD}$ & & \\
\hline \multicolumn{7}{|l|}{ Rt median } \\
\hline Terminal Latency (ms) & 3.93 & 1.36 & 2.60 & 0.42 & 4.26 & $<0.01$ \\
\hline Amplitude $(\mathrm{mV})$ & 6.01 & 1.52 & 7.76 & 0.49 & 4.99 & $<0.01$ \\
\hline Conduction velocity (m/s) & 52.41 & 15.04 & 57.92 & 0.58 & 1.63 & $>0.05$ \\
\hline \multicolumn{7}{|l|}{ Lt Median } \\
\hline Terminal Latency (ms) & 3.09 & 1.33 & 2.58 & 0.35 & 4.32 & $<0.01$ \\
\hline Amplitude $(\mathrm{mV})$ & 5.94 & 1.44 & 7.68 & 0.39 & 5.28 & $<0.01$ \\
\hline Conduction velocity $(\mathrm{m} / \mathrm{s})$ & 52.25 & 14.98 & 57.76 & 0.58 & 1.64 & $>0.05$ \\
\hline \multicolumn{7}{|l|}{$\underline{\text { Rt Ulnar }}$} \\
\hline Terminal Latency (ms) & 3.39 & 1.16 & 2.47 & 0.41 & 3.41 & $<0.01$ \\
\hline Amplitude $(\mathrm{mV})$ & 5.42 & 1.96 & 8.34 & 0.67 & 6.43 & $<0.01$ \\
\hline Conduction velocity $(\mathrm{m} / \mathrm{s})$ & 48.18 & 14.90 & 63.76 & 0.56 & 4.65 & $<0.01$ \\
\hline \multicolumn{7}{|l|}{ Lt Ulnar } \\
\hline Terminal Latency (ms) & 3.36 & 1.10 & 2.53 & 0.35 & 3.27 & $<0.01$ \\
\hline Amplitude (mV) & 5.45 & 0.94 & 8.27 & 0.69 & 11.73 & $<0.01$ \\
\hline Conduction velocity (m/s) & 48.49 & 14.86 & 52.94 & 1.35 & 1.10 & $>0.05$ \\
\hline \multicolumn{7}{|l|}{ Rt Tibial } \\
\hline Terminal Latency (ms) & 4.35 & 2.50 & 4.87 & 0.37 & 0.92 & $>0.05$ \\
\hline Amplitude (mV) & 4.95 & 0.18 & 7.75 & 0.62 & 25.39 & $<0.01$ \\
\hline Conduction velocity $(\mathrm{m} / \mathrm{s})$ & 41.56 & 11.26 & 52.73 & 0.63 & 4.41 & $<0.01$ \\
\hline \multicolumn{7}{|l|}{ Lt Tibial } \\
\hline Terminal Latency (ms) & 4.26 & 2.41 & 4.92 & 0.42 & 1.21 & $>0.05$ \\
\hline Amplitude (mV) & 5.02 & 2.10 & 7.66 & 0.64 & 5.45 & $<0.01$ \\
\hline Conduction velocity $(\mathrm{m} / \mathrm{s})$ & 41.71 & 11.30 & 52.79 & 0.53 & 4.37 & $<0.01$ \\
\hline \multicolumn{7}{|l|}{ Rt Common Peroneal } \\
\hline Terminal Latency (ms) & 3.91 & 2.69 & 2.49 & 0.44 & 1.51 & $>0.05$ \\
\hline Amplitude (mV) & 3.71 & 0.33 & 9.00 & 0.52 & 46.59 & $<0.01$ \\
\hline Conduction velocity $(\mathrm{m} / \mathrm{s})$ & 38.07 & 14.55 & 48.98 & 0.59 & 3.34 & $<0.01$ \\
\hline \multicolumn{7}{|l|}{ Lt Common Peroneal } \\
\hline Terminal Latency (ms) & 3.96 & 2.73 & 2.89 & 0.42 & 1.74 & $>0.05$ \\
\hline Amplitude $(\mathrm{mV})$ & 3.86 & 0.34 & 8.98 & 0.46 & 47.50 & $<0.01$ \\
\hline Conduction velocity (m/s) & 38.18 & 14.59 & 48.89 & 0.49 & 3.27 & $<0.01$ \\
\hline
\end{tabular}


Table 7. Comparison of Sensory Nerve Conduction Study results between OP sprayers and the controls.

\begin{tabular}{|c|c|c|c|c|c|c|}
\hline \multirow{2}{*}{ Sensory NCS Results } & \multicolumn{2}{|c|}{$\begin{array}{c}\text { OP sprayers } \\
\qquad n=36\end{array}$} & \multicolumn{2}{|c|}{$\begin{array}{l}\text { Controls } \\
\qquad \mathbf{n = 2 0}\end{array}$} & \multirow{2}{*}{$\begin{array}{c}\text { Test of } \\
\text { significance } \\
\text { (Student-t } \\
\text { test) }\end{array}$} & \multirow{2}{*}{$\begin{array}{c}\mathbf{P} \\
\text { Value }\end{array}$} \\
\hline & Mean & $\pm \mathrm{SD}$ & Mean & $\pm \mathrm{SD}$ & & \\
\hline \multicolumn{7}{|l|}{$\underline{\text { Rt median }}$} \\
\hline Terminal Latency (ms) & 2.98 & 0.91 & 2.91 & 0.51 & 0.32 & $>0.05$ \\
\hline Amplitude $(\mu \mathrm{V})$ & 28.11 & 1.53 & 31.01 & 1.81 & 6.36 & $<0.01$ \\
\hline Conduction velocity $(\mathrm{m} / \mathrm{s})$ & 54.54 & 10.12 & 55.65 & 11.67 & 0.69 & $>0.05$ \\
\hline \multicolumn{7}{|l|}{ Lt Median } \\
\hline Terminal Latency (ms) & 3.01 & 0.86 & 2.88 & 0.49 & 0.84 & $>0.05$ \\
\hline Amplitude $(\mu \mathrm{V})$ & 28.09 & 1.67 & 30.98 & 1.79 & 6.05 & $<0.01$ \\
\hline Conduction velocity $(\mathrm{m} / \mathrm{s})$ & 53.98 & 11.05 & 54.97 & 11.01 & 1.07 & $>0.05$ \\
\hline \multicolumn{7}{|l|}{$\underline{\text { Rt Ulnar }}$} \\
\hline Terminal Latency (ms) & 3.01 & 0.89 & 2.97 & 0.63 & 2.45 & $<0.05$ \\
\hline Amplitude $(\mu \mathrm{V})$ & 28.50 & 1.83 & 29.55 & 1.01 & 2.37 & $<0.05$ \\
\hline Conduction velocity $(\mathrm{m} / \mathrm{s})$ & 54.39 & 12.09 & 55.68 & 10.98 & 1.43 & $>0.05$ \\
\hline \multicolumn{7}{|l|}{$\underline{\text { Lt Ulnar }}$} \\
\hline Terminal Latency (ms) & 3.10 & 0.99 & 3.03 & 1.02 & 1.51 & $>0.05$ \\
\hline Amplitude $(\mu \mathrm{V})$ & 26.02 & 1.01 & 26.09 & 1.49 & 0.21 & $>0.05$ \\
\hline Conduction velocity $(\mathrm{m} / \mathrm{s})$ & 53.92 & 10.38 & 54.78 & 11.40 & 0.20 & $>0.05$ \\
\hline \multicolumn{7}{|l|}{ Rt Superficial Peroneal } \\
\hline Terminal Latency (ms) & 5.89 & 1.23 & 3.04 & 1.05 & 2.76 & $<0.01$ \\
\hline Amplitude $(\mu \mathrm{V})$ & 21.98 & 4.11 & 25.21 & 1.09 & 3.44 & $<0.01$ \\
\hline Conduction velocity $(\mathrm{m} / \mathrm{s})$ & 50.01 & 10.11 & 55.98 & 12.36 & 4.37 & $<0.01$ \\
\hline \multicolumn{7}{|l|}{ Lt Superficial Peroneal } \\
\hline Terminal Latency (ms) & 6.78 & 1.94 & 2.98 & 1.07 & 2.77 & $<0.01$ \\
\hline Amplitude $(\mu \mathrm{V})$ & 22.55 & 4.53 & 25.89 & 1.10 & 3.23 & $<0.01$ \\
\hline Conduction velocity $(\mathrm{m} / \mathrm{s})$ & 51.16 & 10.13 & 54.98 & 12.04 & 4.81 & $<0.01$ \\
\hline
\end{tabular}


El-Helaly ME. et al.,

Table 8. Prevalence of the diagnosed peripheral neuropathy among the study population.

\begin{tabular}{|l|c|c|c|c|c|c|}
\hline \multirow{2}{*}{$\begin{array}{l}\text { Peripheral } \\
\text { neuropathy }\end{array}$} & \multicolumn{2}{|c|}{$\begin{array}{c}\text { OP sprayers } \\
\mathbf{n = 3 6}\end{array}$} & \multicolumn{2}{c|}{$\begin{array}{c}\text { Controls } \\
\mathbf{n = 2 0}\end{array}$} & $\begin{array}{c}\text { Test of } \\
\text { significance } \\
\text { (Fisher Exact } \\
\text { Test) }\end{array}$ & $\begin{array}{c}\text { P } \\
\text { value }\end{array}$ \\
\cline { 2 - 5 } & $\mathbf{n}$ & $\%$ & $\mathbf{n}$ & $\%$ & & \\
\hline Present & 14 & 38.9 & 1 & 5.0 & & $<0.01$ \\
Absent & 22 & 61.1 & 19 & 95.0 & 7.529 & $<$ \\
\hline
\end{tabular}

Table 9. Classification of the diagnosed peripheral neuropathy among the study population.

\begin{tabular}{|l|c|c|c|c|}
\hline \multicolumn{1}{|c|}{$\begin{array}{c}\text { Classification of the diagnosed } \\
\text { peripheral neuropathies }\end{array}$} & $\begin{array}{c}\text { Subclinical } \\
\text { neuropathy }\end{array}$ & $\begin{array}{c}\text { Possible } \\
\text { neuropathy }\end{array}$ & $\begin{array}{c}\text { Probable } \\
\text { neuropathy }\end{array}$ & $\begin{array}{c}\text { Confirmed } \\
\text { neuropathy }\end{array}$ \\
\hline $\begin{array}{l}\text { Neuropathies among OP sprayers } \\
\text { (n=14):-Mononeuropathy: } 0(0 \%)\end{array}$ & - & - & - & - \\
\hline Polyneuropathy: $14^{\text {a }}(100.0 \%)$ & $11(78.6 \%)$ & $3(21.4 \%)$ & - & - \\
\hline $\begin{array}{l}\text { Neuropathies among controls }(\mathrm{n}=1): \\
\text {-Mononeuropathy: } 1^{\mathrm{b}}(100.0 \%)\end{array}$ & $1(100.0 \%)$ & - & - & - \\
\hline -Polyneuropathy: $0(0.0 \%)$ & - & - & - & - \\
\hline
\end{tabular}

a: all neuropathies were mixed sensory-motor and distributed distal more than proximal.

b: at right median nerve and mixed sensory-motor. 
Table 10. Comparison of the means of AChE levels, age and duration of employment; in presence or absence of neuropathy, among OP sprayers.

\begin{tabular}{|c|c|c|c|c|c|c|}
\hline \multirow{4}{*}{ Variable } & \multicolumn{4}{|c|}{ OP sprayers $(n=36)$} & \multirow{4}{*}{$\begin{array}{c}\text { Tests of } \\
\text { significance }\end{array}$} & \multirow{4}{*}{$\begin{array}{c}\mathbf{P} \\
\text { Value }\end{array}$} \\
\hline & \multicolumn{4}{|c|}{ Neuropathy } & & \\
\hline & \multicolumn{2}{|c|}{$\begin{array}{c}\text { Present } \\
n=14\end{array}$} & \multicolumn{2}{|c|}{$\begin{array}{c}\text { Absent } \\
n=22\end{array}$} & & \\
\hline & Mean & $\pm \mathrm{SD}$ & Mean & $\pm \mathrm{SD}$ & & \\
\hline Plasma AChE ( mU/ml) & 981.7 & 524.9 & 1911.9 & 742.5 & $Z^{*}=3.39$ & $\leq 0.01$ \\
\hline Age (years) & 50.9 & 12.1 & 45.5 & 12.3 & $\mathrm{t}^{*}=1.29$ & $>0.05$ \\
\hline Duration of employment (years) & 11.1 & 4.5 & 8.5 & 3.7 & $\mathrm{t}^{*}=2.01$ & $\leq 0.05$ \\
\hline
\end{tabular}

\section{Disscusion}

A recent review of the literature involving the association between low level exposure to OP esters and peripheral nerve function concluded that peripheral nervous system abnormalities among subjects with current or past exposures to OP esters were mild, inconsistent, and unexplained. Jamal et al. (2002b) reported that the chronic effects on the peripheral and central nervous system of low level exposure, not producing any clinically detectable cholinergic features, are less extensively documented. Many studies have been published on the effects of long-term low level exposure and although most showed correlation between exposure and effect, the results have not been as consistent as those after acute poisoning episodes. Concerning OP exposure assessment in the present study, most of the OP sprayers had practiced OP application with improper personal hygiene, during: storage, mixing and preparation, spraying, disposal, and after-work cleaning up. Moreover, OP sprayers had a lower plasma AChE mean level $(1548.9 \pm 801.7$ $\mathrm{mU} / \mathrm{ml}$ ) compared to the controls $(6751.7 \pm$ $990.8 \mathrm{mU} / \mathrm{ml}$ ) with a highly significant statistical difference. Ogilvie et al. (1992) and O’Malley (1997) reported that pesticide spills, splashes, and immersions resulting from equipment maintenance, spot spraying, mixing and loading have resulted in relatively high pesticide exposure at which long-term adverse health effects can result. The present study did not include any case with history of acute exposure or poisoning 
in the previous month before the study. So, plasma AChE mean levels were measured rather than RBC cholinesterase levels as the depression of the former may be observed at lower exposure doses than those causing depression in RBC cholinesterase (Lotti, 1995).

The present study failed to find any significant difference regarding symptoms and signs of peripheral neuropathy. In consistency, some studies of workers exposed to OP found no relationship of exposure to vibration sensitivity which is a frequently used test to evaluate somato-sensory function (Steenland et al., 2001; Albers et al., 2004).

On the contrary, Stokes et al. (1995) and Cole et al. (1998) reported decreased vibration sensitivity and other signs of peripheral neuropathy, among farmers exposed to OP. Basic neurological examinations, performed by Beach et al. (1996) on sheep dippers showed some signs of sensory disturbance but little evidence of gross motor dysfunction in the farmers. Pilkington et al. (2001) carried out cross sectional exposure-response study of sheep dippers and other non-exposed groups, to investigate the hypothesis that chronic low level exposure to organophosphate (OP) in sheep dips is related to clinically detectable measures of polyneuropathy. The findings showed a strong association between exposure to
OP concentrate and neurological symptoms, but a less consistent association with sensory thresholds. There was only weak evidence of a chronic effect of low dose cumulative exposure to OP. It is suggested that long-term health effects may occur in at least some sheep dippers exposed to OP over a working life, although the mechanisms are unclear.

In the present study, according to neurological examination and NCS results, peripheral neuropathies were found to be more prevalent among OP sprayers (38.9 $\%)$ compared to the controls (5.0\%) with a high statisticaly significant difference $(\mathrm{P}<$ $0.01)$. However, all the diagnosed neuropathies among the OP sprayers were classified as subclinical neuropathies (78.6\%) and possible neuropathies (21.4\%). So, this study failed to diagnose any confirmed neuropathies among OP sprayers. All the diagnosed neuropathies among OP sprayers were mixed sensory - motor axonal polyneuropathies, distributed distal more than proximal. The presence of neuropathy among OP sprayers was associated with significantly lower AChE mean level (981.7 \pm $524.9 \mathrm{mU} / \mathrm{ml}$ ) than those in the absence of neuropathy $(1911.9 \pm 742.5 \mathrm{mU} / \mathrm{ml})$. Also, the neuropathy was found to be related to the duration of employment $(\mathrm{P}<0.05)$ not to the age of OP sprayers. 
Ray (1998) reported that individuals with low levels of the plasma anticholinesterase were vulnerable to the development of persistent chronic neuropathy. In agreement with these results, Amr (1999) found peripheral neuritis among $40 \%$ of a group of three hundred pesticide applicators. $\mathrm{He}$ found polyneuropathy in $36 \%$ of them especially in those exposed for longer durations, more than five years.

In contrast, some studies found no association between chronic low-level exposure to OP and the prevalence of neurological dysfunction (Fiedler et al., 1997; Korsak et al., 1997). Engel et al. (1998) concluded that exposure of farm workers to low levels of OP pesticides during one season was not associated with impaired peripheral neruophysiological function. Albers et al. (2004) found no support for the hypothesis that chronic chlorpyrifos exposure sufficient to produce biological effects on AChE activity was associated with clinically evident or subclinical peripheral neuropathy among chlorpyrifos exposed subjects compared to referents.

Among studies which reported a relationship between chronic low level exposure to OP and peripheral neuropathy, a debate is present regarding the characteristics of the peripheral neuropathy; is it sensory, motor or mixed neuropathy? Jamal et al..$^{13}$ reported that the neuropathy resulting from chronic low exposure to OP, described in their study, was predominantly of a sensory type both symptomatically and neurophysiologically. Bleecker (2007) supposed that, if there was a sensory neuropathy with low dose exposure, it appears to be different from OPIDP. This may be similar to lead neuropathy where motor neuropathy is associated with a high blood lead level ( $>70$ $\mu \mathrm{g}$ per $\mathrm{dL}$ ), whereas at a lower blood lead level (30 $\mu \mathrm{g}$ per $\mathrm{dL})$ only sensory changes are found. Mechanistic studies have suggested that long-term effects of Organophosphate may be mediated by cholinergic mechanisms or phosphorylation of neuronal protein sites, and other mechanisms may be important as genetic differences in detoxification enzymes and nonspecific binding which may account for some of the variations between people in susceptibility to anticholinesterases. It is probable, therefore, that the development of long-term health effects has a genetic component but susceptible people will be difficult to identify without a very large study population, good comparative exposure data, and knowledge of the toxic mechanism (Blain, 2001). The present study did not find any confirmed neuropathy among OP sprayers, however $78.6 \%$ of all diagnosed neuropathies were subclinical. This may reflect an early stage of neuropathy as Blake (2004) 
stated that toxic axonopathy can manifest as defects in sensory or motor functions, or a combination of the two. For most neurotoxicants, sensory changes are noticed first, followed by progressive involvement of motor neurons.

A limited number of studies considering neurological health effects of long-term low dose exposures to OP have often only reported findings in small groups of subjects and have relied on basic assessments of neurological function. The nature of the exposure varies considerably between different studies, as does the presence of significant co-exposures, and the availability of adequate personal protection. This makes it difficult to overview the findings of most published studies in a consistent manner (Jamal et al., 2002b).

At present, there is inadequate information from epidemiological studies to determine whether adverse neurological effects result from low-level exposure to OP (Albers et al., 2004). The limitations of the present study were the small sample size and the fact that we did not assess the individual exposure to each type of OP nor did we calculate the cumulative exposure to OP used in this study. Also we did not use Quantitative Sensory Testing (QST) both with NCS as to monitor the neuropathic effects both on small and long nerve fibers. We recommend overall reduction and legislative control of the pesticide use and disposal, encouraging OP sprayers to use the protective equipment, adoption of safety practices during field work and implementing health education programs about the risks of pesticide exposure. Serial measurement of AChE levels, occupational health surveillance and periodic medical monitoring with emphasis on the peripheral nervous system are recommended for all OP sprayers. More research is still needed on a large scale to explore the health effects of chronic exposure to OP and other pesticides, specially on the nervous system.

\section{Conclusions:}

Most of OP sprayers had improper work practice and most of them did not use any protective equipment, increasing their exposure to OP. This study reported a higher prevalence of peripheral neuropathies among OP sprayers in comparison to the controls $(\mathrm{P}<0.01)$, however, most of the diagnosed neuropathies among the OP sprayers were subclinical neuropathies. The study failed to diagnose any confirmed neuropathies among OP sprayers. The picture of the diagnosed neuropathy was mixed sensory - motor axonal polyneuropathy, distributed distal more than proximal and associated with lower levels of AChE and longer duration of employment. These re- 
sults may denote an early stage of neuropathy and follow up study is needed to evaluate progress in the neuropathic effect of OP among those workers.

\section{Acknowledgements}

We thank Mr. Mahmoud El Sherbeeny for producing the information concerning OP pesticides used by the OP sprayers in the present study.

\section{References}

1. Albers JW, Garabrant DH, Schweitzer SJ, et al. (2004): The effects of occupational exposure to chlorpyrifos on the peripheral nervous system: a prospective cohort study. Occup Environ Med. 61:201-11.

2. Amr, MM (1999): Pesticide monitoring and its health problems in Egypt, a Third World country. Toxicol Lett. 107(1-3):1-13.

3. Beach JR, Spurgeon A, Stephens R, et al. (1996): Abnormalities on neurological examination among sheep farmers exposed to organophosphate pesticides. Occup Environ Med. 53:520-25.

4. Blain PG (2001): Adverse health effects after low level exposure to Organophosphate. Occup Environ Med. 58:689-90.

5. Blake BL (2004): Toxicology of the Nervous System. In: Hodgson E, ed. A Textbook Of Modern Toxicology ( $3^{\text {rd }}$ ed.). John Wiley and Sons. 279-97.

6. Bleecker ML (2007): Toxic peripheral neuropathy. In: Rom WN, ed. Environmental and Occupational Medicine (4thed.). Lippincott Williams and Wilkins. 641-55.
7. Cole DC, Carpio F, Julian J and leon N (1998): Assessment of peripheral nerve function in an Ecuadorian rural population exposed to pesticides. J Toxicol Environ Health. 55:77-91.

8. Cope WG, Leidy RB and Hodgson E (2004). Organophosphorus insecticides. In: Hodgson E, ed. A textbook of modern toxicology ( $3^{\text {rd }}$ ed.). John Wiley and Sons. 58-60.

9. Dyck PJ, Litchy WJ and Lehman KA (1995): Variables influencing neuropathic endpoints: the Rochester Diabetic Neuropathy Study of Healthy Subjects. Neurology. 45:1115-21.

10. Ellman GL, Courtney KK and Andres WJ (1961): A new and rapid colorimetric determination of acetylcholinesterase activity. Biochem Pharmacol. 7:88-95.

11. Engel LS, Keifer MC, Checkoway H, et al. (1998): Neurophysiological function in farm workers exposed to organophosphate pesticides. Arch Environ Health. 53(1):7-14.

12. Fiedler N, Kipen H, Kelly-McNeil K, et al. (1997): Long-term use of Organophosphate and neuro-psychological performance. Am J Ind Med. 32:227-86.

13. Jamal GA, Hansen S and Julu PO (2002a): Low level exposures to organophosphorus esters may cause neurotoxicity. Toxicology.181-182:23-33.

14. Jamal GA, Hansen S, Pilkington A, et al. (2002b): A clinical neurological, neurophysiological, and neuropychological study of sheep farmers and dippers exposed to organophosphate pesticides. Occup Environ Med. 59:434-41.

15. Kamel F, Engel LS, Gladen BC, et al. (2005): Neurologic symptoms in licensed private pesticide applicators in the agricultural health study. Environ Health Perspect. 113(7):877-82. 
16. Kamel, F, Tanner C, Umbach D, et al. (2007): Pesticide exposure and self-reported Parkinson's disease in the agricultural health study. Am J Epidemiol. 165(4):364 -74.

17. Korsak RJ, Sato MM (1997): Effects of chronic organophosphate pesticide exposure on the central nervous system. Clin Toxicol. 11:83-95.

18. Lotti M (1995): Cholinesterase inhibition: complexities in interpretation. Clin Chem. 41:181418.

19. Miranda J, McConnell R, Wesseling C, et al. (2004): Muscular strength and vibration thresholds during two years after acute poisoning with organophosphate insecticides. Occup Environ Med.6:e4.

20. Moretto A and Lotti M (1998): Poisoning by organophosphorus insecticides and sensory neuropathy. J Neurol Neurosurg Psychiatry. 64:463468.

21. Niven KM, Scott AJ and Hagen S (1993): Occupational hygiene assessment of sheep dipping practices and processes. Edinburgh: Institute of Occupational Medicine. IOM report TM/93/03:1993.

22. Ogilvie L, Kross BC, Popendorf WJ, et al. (1992): Summary report: Assessment methods for pesticide exposure (AMPE) study. In: NCI Progress Report, Institute of Agriculture Medicine and Occupational Health. Iowa: University of Iowa:50.
23. Oh SJ (2003): Nerve Conduction in Focal Neuropathies. In: Oh SJ, ed. Clinical electromyography and nerve conduction studies ( $3^{\text {ed }}$ ed.). Lippincott Wiliams and Wilkins; Philadelphia:62332.

24. O'Malley M (1997): Clinical evaluation of pesticide exposure and poisoning. Lancet. 349:116166.

25. Pilkington A, D Buchanan, G A Jamal, et al. (2001): An epidemiological study of the relations between exposure to organophosphate pesticides and indices of chronic peripheral neuropathy and neuropsychological abnormalities in sheep farmers and dippers. Occup Environ Med. 58:702-10.

26. Ray DE (1998): Chronic effects of low level exposure to anticholinesterases - a mechanistic review. Toxicol Lett. 103:527-33.

27. Steenland K, Dick RB, Howell RJ, et al. (2001): Neurologic function among termiticide applicators exposed to chloropyrifos. Environ Health Perspect. 108:293-300.

28. Stokes L, Stark A, Marshall E, et al. (1995): Neurotoxicity among pesticide applicators exposed to Organophosphate. Occup Environ Med. 52:648-53.

29. WHO (2004). The WHO recommended classification of pesticides by hazard and guidelines to classification: 2004. World Health Organization. ISBN 9241546638. 